\title{
Diagnostic value of medical thoracoscopy for undiagnosed pleural effusions
}

\author{
RUI-LIN CHEN，YONG-QING ZHANG，JUN WANG，HUA WU and SHU-MEI YANG \\ Department of Respiratory Medicine, Shaanxi Provincial People's Hospital, Xi'an, Shaanxi 710068, P.R. China
}

Received March 14, 2017; Accepted July 20, 2017

DOI: $10.3892 /$ etm.2018.6742

\begin{abstract}
To investigate the diagnostic value of medical thoracoscopy for the diagnosis of undiagnosed pleural effusions, a retrospective study was performed on the clinical data of 86 patients with undiagnosed pleural effusions who had medical thoracoscopy at Shaanxi Provincial People's Hospital (Xi'an, China) between May 2012 and November 2013. Of the 86 patients, 79 cases of pleural effusions were confirmed by medical thoracoscopic biopsy with a diagnosis rate of $91.9 \%$. In these 79 confirmed patients, 37 had pleura cancer metastasis (43.0\%) and 20 had tuberculous pleuritis (23.3\%). The most common type of malignant tumor was lung cancer, accounting for $86.5 \%$ of the patients with pleural metastasis. Tuberculosis was often observed in the 16-35 years of age patient group, while malignant tumors were typically detected in the 36-65 year and 65 years and above patient groups. Notably, the overall diagnosis distribution had little connection with sex or smoking-history. Neither mortality nor major complications were observed in patients who received medical thoracoscopy examination. In conclusion, medical thoracoscopy is a safe and effective examination method and has an important diagnostic value for unidentified pleural effusion in patients.
\end{abstract}

\section{Introduction}

The diagnosis of unidentified pleural effusions is one of the most difficult and complicated tasks in respiratory medicine (1). Prior to the application of thoracoscopy, hydrothorax exfoliative cytologic examination and closed pleural biopsy were the two methods typically used; however, these methods have a low positive diagnostic rate (2). The clinical application of thoracoscopy thus provides a novel diagnostic method for undiagnosed pleural effusions. Compared to conventional closed pleural biopsy, the thoracoscopy has notable advantages. It overcomes the blindness of closed pleural biopsy and

Correspondence to: Professor Shu-Mei Yang, Department of Respiratory Medicine, Shaanxi Provincial People's Hospital, 256 West Friendship Road, Xi'an, Shaanxi 710068, P.R. China

E-mail: halcyonsea@tom.com

Key words: medical thoracoscopy, pleural effusion, diagnosis markedly improves diagnostic accuracy of pleural effusions, and therefore improves the positive diagnostic rate of pleural diseases (3-5). It has been reported that the overall positive diagnostic rate with thoracoscopy may reach $71-100 \%$ (6-8). For example, a retrospective study by Hansen et al (9) investigated the diagnostic value of thoracoscopy in 146 patients with pleural effusions, and the positive diagnostic rate reached 90.4\%. Among these patients, 136 had negative results through three cytological and microbiological examinations (9).

The present retrospective study included 86 patients with pleural effusions who received medical thoracoscopy examination at Shaanxi Provincial People's Hospital (Xi'an, China). A diagnosis rate of $91.9 \%$ was identified and it was demonstrated that malignant tumors were the most common cause of pleural effusion in the 36-65 and 65 years and above age groups, while tuberculous pleuritis was the most common cause in the 16-35-year-old age group. Sex and smoking-history had little impact on diagnosis distribution.

\section{Patients and methods}

Patients. A retrospective study was conducted on clinical data of 86 patients (50 males and 36 females; age range, 17-84 years; mean, $58 \pm 15$ years) with pleural effusions who received medical thoracoscopy in the Endoscopy Center of Shaanxi Provincial People's Hospital between May 2012 to November 2013. The indication of medical thoracoscopy was undiagnosed pleural effusion, which referred to the situation that the cause of disease could not be identified through routine examination of pleural fluid, biochemical tests, bacteriology, exfoliative cytology as well as closed pleural biopsy examination. No contraindication had been demonstrated for any patients receiving medical thoracoscopy. The procedure was approved by the Ethics Committee of Shaanxi Provincial People's Hospital. All patients and family members were informed of the surgical procedure and signed an informed consent form prior to the procedure.

Medical thoracoscopy procedure. A bent-type electronic thoracoscope (Olympus BF-240) with EVIS-260 light-source and video camera system (Olympus Corporation, Tokyo, Japan) was used for the thoracoscopy procedure. Routine blood tests, bleeding and clotting time tests, and electrocardiograms were performed prior to examination. Artificial pneumothorax on the affected side was set up prior to surgery. The whole 
procedure was conducted in the endoscope room. Heart rate, breathing frequency, blood pressure, oxygen saturation and consciousness were closely monitored. Each patient was placed in a lateral decubitus position with the affected side up. The thoracoscope was inserted into the pleural cavity at the midpoint between the 6 and 7 th ribs along the midaxillary line or axillary line, through a 1-2-cm cut under local anesthesia. If there was no pleural adhesion observed, the trocar was inserted vertically and put into the thoracoscope. The pleural effusion was examined and drawn out as much as possible to obtain satisfactory exposure. The changes of parietal and visceral pleura were examined carefully. If abnormal pleural nodules, pleural congestion, thickening, adhesion and ulcers were observed under the thoracoscope, 5-10 pieces of tissue were collected under direct vision via a protractor. Following surgery, all fluid and air inside the pleural cavity were drawn out as much as possible and the closed drainage trocars was left inside. Diagnosis of tumor, tuberculosis or other pleural lesions was performed according to histological and cytological changes, as previously described in the British thoracic society guidelines (10). Histological analysis was performed following standard protocol. In brief, tissue samples were fixed in $10 \%$ neutral buffered formalin overnight at room temperature and subsequently embedded in paraffin blocks. Sections were cut to $6-\mu \mathrm{m}$ thick and stained with a commercial hematoxylin and eosin staining kit (haematoxylin for $4 \mathrm{~min}$ and eosin for $2 \mathrm{~min}$, at room temperature). Slides were examined under standard bright-field microscope with a x10 or x 20 object lens.

\section{Results}

Medical thoracoscopy examination delivers a high diagnosis rate. Among the 86 patients with undiagnosed pleural effusions, 50 were males and 36 were females. Their age range was $17-84$ years with an average of $58 \pm 15$ years. Among them, 2 patients had pleural effusion with a little peritoneal effusion, and 6 patients had pleural effusion with a small amount of pericardial effusion. Clinical features of these patients were summarized in Table I. The thoracoscopy examination took 15-120 min with an average of $35 \mathrm{~min}$. In 86 patients receiving thoracoscopy, 79 were given clear diagnosis and 5 remained undiagnosed. Examination failed only in 2 patients: 1 patient had their diaphragm muscle obviously moved up and the thoracoscope went into peritoneal cavity by mistake due to pleural adhesions; the other patient had serious pleural thickening and adhesion making it difficult for the thoracoscope to enter the pleural cavity for observation. Altogether, the positive diagnosis rate was $91.9 \%(79 / 86)$.

Among the 86 patients receiving thoracoscopy, the largest group of patients were diagnosed with pleura cancer metastasis $(37 / 86 ; 43.0 \%)$, the second largest group of patients were diagnosed with tuberculous pleuritis $(20 / 86 ; 23.3 \%)$, while third largest group of patients was diagnosed with non-specific pleuritis $(13 / 86 ; 15.1 \%)$. The detailed results of the pathological examination were demonstrated in Table II. Among the 13 patients with non-specific pleuritis, 6 were considered as tuberculous pleuritis and given tentative anti-tuberculosis treatment following pleural effusion absorption. In 1 suspicious patient, it was confirmed that they did not have pleural mesothelioma at the 6-month follow-up visit
Table I. Patient features of the study group $(n=86)$.

Average value or

Clinical features number of cases

Age, years

$58.0 \pm 15.0(17-84)^{a}$

Sex (male/female)

$50 / 36$

Position of pleural effusion

$36 / 40 / 10$

(left/right/both sides)

Smoker/non-smoker

$48 / 38$

${ }^{\mathrm{a}}$ Data are presented as the mean \pm standard deviation (range).

Table II. Results of pleura pathological examination for 86 patients with pleural effusions.

\begin{tabular}{lc}
\hline Pathological results & $\mathrm{n}(\%)$ \\
\hline Pleura cancer metastasis & $37(43.0)$ \\
Lung adenocarcinoma metastasis & $26(30.2)$ \\
Lung squamous cell cancer metastasis & $6(6.9)$ \\
Breast cancer metastasis & $1(1.1)$ \\
Alimentary canal tumor metastasis & $2(2.3)$ \\
Malignant tumor source unidentified & $2(2.3)$ \\
Pleural mesothelioma & $3(3.5)$ \\
Tuberculous pleuritis & $20(23.3)$ \\
Non-specific pleuritis & $13(15.1)$ \\
Parapneumonic effusion & $4(4.7)$ \\
Secondary fetal hydrothorax after & $1(1.1)$ \\
pneumothorax & $1(1.1)$ \\
Secondary fetal hydrothorax of & \\
chronic cholecystitis & $5(5.8)$ \\
Undiagnosed by pathology & $2(2.3)$ \\
Failed examination &
\end{tabular}

for pleural effusion absorption. Furthermore, 1 patient with a liver abscess, pneumonia and pleural effusion, was considered as parapneumonic effusion and given anti-infective treatment following effusion absorption. Among the 5 patients whose diseases were not clearly diagnosed, 1 was diagnosed later on with poorly differentiated adenocarcinoma from bilateral ovaries; 1 was diagnosed with immature teratoma through an exploratory laparotomy; 1 was diagnosed with malignant schwannoma through a thoracotomy and pathological analysis; the remaining 2 patients remained undiagnosed (data not shown).

Different causes of pleural effusion present different manifestations under the thoracoscope. A thorough understanding of these manifestations will substantially improve the positive diagnostic rate of biopsy. Malignant tumor metastasis pleural effusions (Fig. 1) were predominantly bloody pleural fluid, and there were numerous grey-white nodules or lumps with different sizes on parietal and visceral pleura, which had a well-defined boundary and friable nature and were relatively 

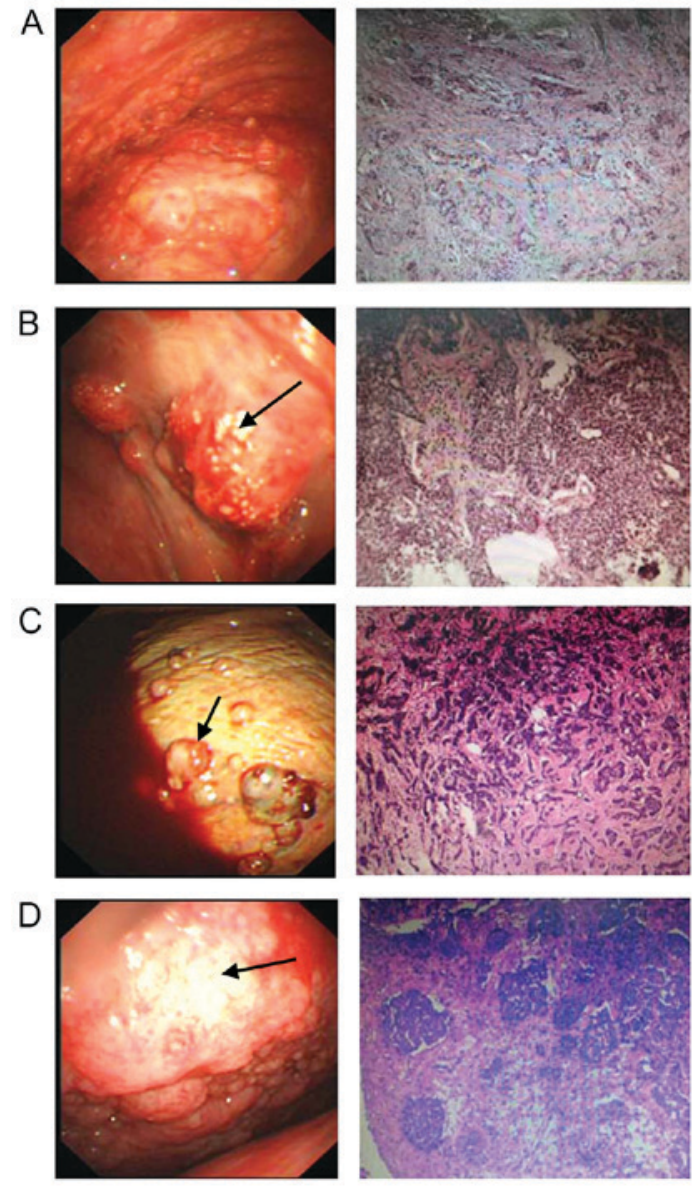

Figure 1. Patients with pleural effusion diagnosed with malignant tumors. (A) Lung adenocarcinoma metastasis. (B) Lung squamous cell cancer metastasis. (C) Breast cancer metastasis. (D) Pleural mesothelioma. Images are thoracoscopy view (500K CCD, Olympus, left panel) and pathological section view (hematoxylin and eosin staining; magnification, x50, right panel). For metastatic tumors of pleura, diffuse or solitary pulmonary nodules or lumps with different shapes and sizes were commonly observed on the pleural wall or diaphragmatic pleura. Some nodules fused into a lump (B, black arrow). Pleural mesothelioma was associated with bloody pleural fluid and widely distributed ball-shaped nodules (C, black arrow) or lumps with different sizes on parietal pleura. Pleura congestion and thickening ( $\mathrm{D}$, black arrow) were also found.

easy to biopsy. Additionally, the nodules or lumps were prone to bleed following biopsy (Fig. 1A-C). For tuberculous pleuritis, the pleura was observed with obvious congestion and edema, and hatpin-sized grey-white or light-yellow nodules were often seen scattering or gathering on the pleura, accompanied with a lot of fibrin and cord-like adhesions (Fig. 2). The pleural mesothelioma was manifested by numerous lumpy or flat lesions on the parietal pleura, and they did not tend to bleed following biopsy (Fig. 1D). The non-specific pleuritis was manifested with diffusive or limited congestion, edema and thickening on parietal pleura (Fig. 3). Overall, we recommend selecting fragile and rubbery tissue lesions for biopsy, and testing as many lesions as possible to avoid incorrect diagnosis.

Diagnosis distribution of pleural effusions is linked to age but not sex or smoking-history. The undiagnosed pleural effusions had different clinical causes at different ages (Table III). In the present study, tuberculosis was often observed in 16-35-year-old patients $(13 / 23 ; 56.5 \%)$, while malignant

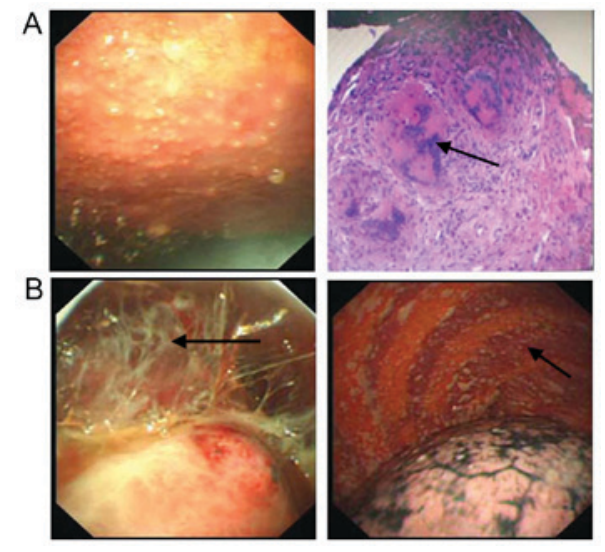

Figure 2. Patients with pleural effusion diagnosed with tuberculous pleuritis. (A) Acute stage. Images are thoracoscopy view (500K CCD, Olympus, left penal) and pathological section view (hematoxylin and eosin staining; magnification, x50, right panel). (B) Chronic stage (500K CCD, Olympus, both panels). Tuberculous pleuritis was often diagnosed based on diffuse congestion and thickening of the pleural wall or diaphragmatic pleura, with miliary and popular nodules. At the chronic stage, fibrous adhesions (B, black arrow) were often observed while caseating sphacelus (A, black arrow) was occasionally seen.

tumors became the major cause in 36-65-year-old patients $(24 / 35 ; 68.6 \%)$ and patients who were 65 years old and above $(8 / 12 ; 66.7 \%)$. Among the malignant tumors, lung cancer with pleural metastasis was the most common type (32/37; 86.5\%), and the majority of these patients had metastatic lung adenocarcinoma (data not shown).

Notably, smoking history and sex had no notable impact on the distribution of diagnosis. It is well known that lung cancer is closely related to cigarette smoking (11); however, the diagnosis of malignant tumors was only slightly higher in smokers than non-smokers (56.1 vs. $48.3 \%$, respectively). This may be due to the fact that secondhand smoke has been a severe social problem in China in past years (12). Similarly, the diagnosis of malignant tumors was also slightly higher in males than females (61.9 vs. $39.3 \%$, respectively), given that the population of male smokers is much higher than female smokers in China (13). However, a larger sample size is required to reach a conclusion with statistical significance.

Potential complications of thoracoscopy examination. The most common complication following thoracoscopy examination was chest pain $(47 / 86 ; 54.7 \%)$. Other complications included subcutaneous emphysema (16 cases accounting for $18.6 \%$ ), pleural hemorrhage (8 cases accounting for $9.3 \%$ ), low-grade fever (5 cases accounting for 5.8\%), light nausea (4 cases accounting for 4.7\%) and re-expansion pulmonary edema ( 1 case accounting for 1.2\%). All symptoms disappeared within 1-3 days, and no mortality occurred (data not shown).

Although medical thoracoscopy is considered as a minimally invasive procedure, it is still possible to cause intraoperative hemorrhage, re-expansion pulmonary edema, secondary infection, chest pain, fever and other complications. Among the 84 patients receiving the thoracoscopy procedure in our facility, only 1 developed re-expansion pulmonary edema following surgery, and this was relieved quickly under standard care. Aside from that, the most common complications 
Table III. Diagnosis distribution of patients with pleural effusions to three major categories (age, sex and smoking history).

\begin{tabular}{|c|c|c|c|c|}
\hline \multirow[b]{2}{*}{ Group } & \multicolumn{4}{|c|}{ Diagnosis } \\
\hline & Malignant tumor, n (\%) & Tuberculosis, n (\%) & Non-specific pleuritis, n (\%) & Total $(\%)$ \\
\hline \multicolumn{5}{|l|}{ Age, years } \\
\hline $16-35$ & $5(21.7)$ & $13(56.5)$ & $5(21.7)$ & $23(100.0)$ \\
\hline $36-65$ & $24(68.6)$ & $5(14.3)$ & $6(17.1)$ & $35(100.0)$ \\
\hline 65 and above & $8(66.7)$ & $2(16.7)$ & $2(16.7)$ & $12(100.0)$ \\
\hline \multicolumn{5}{|l|}{ Smoking history } \\
\hline Smoker & $23(56.1)$ & $12(29.3)$ & $6(14.6)$ & $41(100.0)$ \\
\hline Non-smoker & $14(48.3)$ & $8(27.6)$ & $7(24.1)$ & $29(100.0)$ \\
\hline \multicolumn{5}{|l|}{ Sex } \\
\hline Male & $26(61.9)$ & $11(26.2)$ & $5(11.9)$ & $42(100.0)$ \\
\hline Female & $11(39.3)$ & $9(32.1)$ & $8(28.6)$ & $28(100.0)$ \\
\hline Total (\%) & $37(52.9)$ & 20 (28.6) & $13(18.6)$ & $70(100.0)$ \\
\hline
\end{tabular}

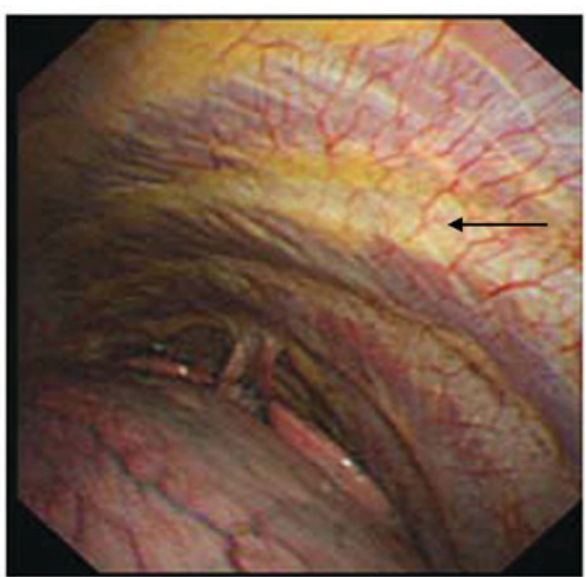

Figure 3. Patients with pleural effusion diagnosed with non-specific pleuritis. Image is the thoracoscopy view (500K CCD, Olympus). Diffuse or limited congestion, edema and thickening on the parietal pleura was often observed (black arrow).

were chest pain, often found following pleural atresia surgery, as well as fever (mostly low-grade fever), which disappeared within 1-3 days without any special treatment (9).

\section{Discussion}

Pleural effusion is a clinical feature of various diseases, and currently, it is sometimes difficult to diagnose the causes of clinical pleural effusions (1). There are $20-25 \%$ of cases whose causes cannot be determined with the existing routine methods (10). The medical thoracoscopy developed in recent years is an invasive surgical technique that may be accomplished independently by a pulmonary physician under local anesthesia. Research has demonstrated that medical thoracoscopy is able to markedly increase the positive diagnostic rate of undiagnosed pleural effusions (3). The present retrospective study included the clinical data of 86 patients with undiagnosed pleural effusions who had received medical thoracoscopy, in order to investigate the diagnostic value of medical thoracoscopy in diagnosis of undiagnosed pleural effusions. The diagnosis rate of thoracoscopy in the present study reached as high as $91.9 \%$, which is comparable with what has been reported in previous studies (6-8).

Malignant tumors represent the top cause of clinical pleural effusions in general, and lung adenocarcinoma is frequently observed in these patients (14). However, there is regional discrepancy regarding the primary non-malignant cause, particularly in terms of tuberculous pleuritis (15). Tuberculosis is the third most common cause of pleural effusions in Spain, while it is not a major cause in the United States (16). In the present study, tuberculosis was diagnosed in $23.3 \%$ of the patients following thoracoscopy. The early diagnosis made it possible for us to apply pleural drainage at the acute stage of tuberculous pleuritis, which effectively prevented chronic changes and reduced the length of hospitalization. At the chronic stage, such as separated and encircled pleura, we used biopsy forceps to remove the adhesive band, sphacelus, as well as encapsulated effusion resulting from earlier clamping, thus improving the therapeutic effect.

In the present study, malignant tumors and tuberculous pleuritis accounted for 43.0 and $23.3 \%$ of the causes of undiagnosed pleural effusions, respectively. These are also the two most common causes of pleural effusions in China (10). The third cause was non-specific pleuritis, which accounted for $15.1 \%$ of cases and, in these patients, tuberculous pleuritis was frequently found at the 6-18-month follow-ups. A study by Venekamp et al (17) also reported that, from 75 patients who were diagnosed with non-specific pleuritis under thoracoscopy in $2005,91.77 \%$ had a benign process and only $8.3 \%$ evolved into tumor according to a 3 -year follow-up study. Thus, when a patient is diagnosed with non-specific inflammation under thoracoscopy, the clinician should still conduct dynamic observation and whole-body screening to determine the cause.

Pleural mesothelioma is a rare tumor that originates from pleural mesothelium tissue or sub-pleural interstitial tissue (18). The positive diagnostic rate is usually low by a pleural effusion cytologic examination, and it has been reported to be only $0-22 \%$ (19). This is likely due to the fact that there are very few 
mesothelial cells falling off in pleural effusion and sometimes it is difficult to conduct a qualitative analysis with a single cell; or it is because the cell has been kept in pleural effusion for a long time and has lost its features due to degeneration, therefore it is not easily distinguished from normal mesothelial cells (18). The closed pleural biopsy has a positive diagnostic rate of $60 \%$ because the lesion of pleura is not evenly distributed (18). With thoracoscopy, it is possible to examine the whole pleural cavity to discover early lesions and conduct biopsy under visual inspection, therefore the positive diagnostic rate of pleural mesothelioma may reach $91-100 \%$ (20). Thus, it is necessary to conduct thoracoscopy as early as possible for patients with undiagnosed pleural effusions.

In conclusion, medical thoracoscopy is a useful tool for diagnosing pleural effusions with unidentified causes, and it markedly improves the positive diagnostic rate of pleural effusions. This procedure has the advantages of visual inspection, easy procedure, high safety and few complications, and has an important application value for the diagnosis of pleural effusions with unidentified causes, particularly for pleural metastatic tumors, tuberculous pleuritis and pleural mesothelioma.

\section{References}

1. McGrath EE and Anderson PB: Diagnosis of pleural effusion: A systematic approach. Am J Crit Care 20: 119-128, 2011.

2. Prakash UB and Reiman HM: Comparison of needle biopsy with cytologic analysis for the evaluation of pleural effusion: Analysis of 414 cases. Mayo Clin Proc 60: 158-164, 1985.

3. Kendall SW, Bryan AJ, Large SR and Wells FC: Pleural effusions: Is thoracoscopy a reliable investigation? A retrospective review. Respir Med 86: 437-440, 1992.

4. Rodriguez-Panadero F, Janssen JP and Astoul P: Thoracoscopy: General overview and place in the diagnosis and management of pleural effusion. Eur Respir J 28: 409-422, 2006.

5. Tassi GF and Tschopp JM: The centenary of medical thoracoscopy. Eur Respir J 36: 1229-1231, 2010.

6. Sakuraba M, Masuda K, Hebisawa A, Sagara Y and Komatsu H: Diagnostic value of thoracoscopic pleural biopsy for pleurisy under local anaesthesia. ANZ J Surg 76: 722-724, 2006.
7. Blanc FX, Atassi K, Bignon J and Housset B: Diagnostic value of medical thoracoscopy in pleural disease: A 6-year retrospective study. Chest 121: 1677-1683, 2002.

8. Lee P, Hsu A, Lo C and Colt HG: Prospective evaluation of flex-rigid pleuroscopy for indeterminate pleural effusion: Accuracy, safety and outcome. Respirology 12: 881-886, 2007

9. Hansen M, Faurschou P and Clementsen P: Medical thoracoscopy, results and complications in 146 patients: A retrospective study. Respir Med 92: 228-232, 1998.

10. Maskell NA and Butland RJ; Pleural Diseases Group, Standards of Care Committee, British Thoracic Society: BTS guidelines for the investigation of a unilateral pleural effusion in adults. Thorax 58 (Suppl 2): ii8-i17, 2003.

11. Murray JF and Mason RJ: Murray and Nadel's textbook of respiratory medicine. Saunders/Elsevier, Philadelphia, PA, 2010.

12. Taylor R, Najafi F and Dobson A: Meta-analysis of studies of passive smoking and lung cancer: Effects of study type and continent. Int J Epidemiol 36: 1048-1059, 2007.

13. Li Q, Hsia J and Yang G: Prevalence of smoking in China in 2010. N Engl J Med 364: 2469-2470, 2011.

14. Boutin C, Viallat JR, Cargnino P and Farisse P: Thoracoscopy in malignant pleural effusions. Am Rev Respir Dis 124: 588-592, 1981.

15. Porcel JM and Vives M: Differentiating tuberculous from malignant pleural effusions: A scoring model. Med Sci Monit 9: CR175-CR180, 2003.

16. Baumann MH, Nolan R, Petrini M, Lee YC, Light RW and Schneider E: Pleural tuberculosis in the United States: Incidence and drug resistance. Chest 131: 1125-1132, 2007.

17. Venekamp LN, Velkeniers B and Noppen M: Does 'idiopathic pleuritis' exist? Natural history of non-specific pleuritis diagnosed after thoracoscopy. Respiration 72: 74-78, 2005.

18. Barreiro TJ and Katzman PJ: Malignant mesothelioma: A case presentation and review. J Am Osteopath Assoc 106: 699-704, 2006.

19. Renshaw AA, Dean BR, Antman KH, Sugarbaker DJ and Cibas ES: The role of cytologic evaluation of pleural fluid in the diagnosis of malignant mesothelioma. Chest 111: 106-109, 1997.

20. Boutin C, Rey F, Gouvernet J, Viallat JR, Astoul P and Ledoray V: Thoracoscopy in pleural malignant mesothelioma: A prospective study of 188 consecutive patients. Part 1: Diagnosis. Cancer 72: 394-404, 1993

This work is licensed under a Creative Commons Attribution-NonCommercial-NoDerivatives 4.0 International (CC BY-NC-ND 4.0) License. 